\title{
La imagen de la Marca España en las redes a través de su campaña "I need Spain"
}

\author{
Juan Manuel CoRbacho VALENCIA \\ Universidad de Vigo \\ jmcorbacho@uvigo.es \\ Mónica Valderrama SAntomé \\ Universidad de Vigo \\ santome@uvigo.es \\ Silvia GARCía MiróN \\ Universidad de Vigo \\ silviamiron@uvigo.es
}

\begin{abstract}
Resumen:
Al igual que las marcas comerciales, también los países se diferencian por las asociaciones percibidas y proyectadas hasta el punto que resultan medibles a través de distintos estudios. El presente trabajo aborda una de las últimas campañas realizadas para fomentar la marca España ante distintos públicos, principalmente externos. Se trata de "I need Spain" que se orquestó a través de medios digitales con distintas plataformas. Se realiza una reflexión crítica acerca de los agentes implicados en dicha tarea y las perspectivas de futuro al respecto.
\end{abstract}

Palabras clave: Imagen de marca país; internacionalización; gestión de marca; medios digitales.

\section{The nation brand of Spain on the internet through the "I need Spain" campaign}

\begin{abstract}
:
Both commercial and nation brands get differentiated by perceived and broadcasted associations and perceptions that are measureable through different reports. This paper focusses on the last campaign to foster Spain's nation brand between different target audiences, mainly from abroad. The campaign titled "I need Spain" has run through digital media and different platforms. The authors analyze the role played by the agents involved and future perspectives on this issue.
\end{abstract}

Key Words: Nation brand; internationalisation; brand management; digital media.

\section{Referencia normalizada:}

Corbacho Valencia, J.M, Valderrama Santomé, M. y García Mirón, S. (2014): La imagen de la marca España en las redes a través de su campaña "I need Spain". Historia y Comunicación Social. Vol. 19. Núm. Especial Enero. Págs. 501-512.

Sumario: 1.Introducción: la imagen de marca país en el contexto actual. 2. Metodología. 3.La gestión de la marca. 4. Las dimensiones de la imagen de marca país y su medición. 5. La gestión de la marca país España. 6. Análisis de la campaña "I need Spain". 7. Conclusiones. 8. Bibliografía. 


\section{Introducción: la imagen de marca país es el contextos actual}

Venimos observando de un tiempo a esta parte que todo tipo de noticias del ámbito político, social, económico, cultural, etc. se interpretan desde la óptica de su influencia en la marca país. En esta larga lista no solo se incorporan todos los éxitos deportivos cosechados por las distintas selecciones y deportistas de fútbol, balonmano, baloncesto, tenis, Fórmula 1, las distintas categorías de motociclismo y un largo etcétera como sinónimo de trabajo en grupo y superación. No obstante, otras noticias parecen ensombrecer estos logros, caso de los conflictos derivados de supuestos casos de corrupción de la clase política y de miembros de la familia real, la crisis galopante, eso sí en pleno proceso de recuperación, y convulsiones internas como la cuestión catalana, entre otras. Tanto es así, que incluso la reciente catástrofe de Angrois fue interpretada en términos de marca país en lo que a los trenes de alta velocidad se refiere, uno de los buques insignia de la ciencia e innovación española, junto con el trasplante de órganos. Echando la vista atrás dicha, podemos destacara el reportaje publicado por Samuel Aranda en el New York Times titulado "Spain Recoils as Its Hungry Forage Trash Bins for a Next Meal" (España retrocede a medida que su hambre le obliga a buscar la siguiente comida en los cubos de basura ) el 24 de septiembre de 2012, con unas fotos más que llamativas de manifestaciones y personas buscando en contenedores. Según El País (9 de septiembre de 2013) "La marca España cotiza a la baja" en alusión a que Madrid nuevamente no consiguiera convertirse en sede de los Juegos Olímpicos 2020.

Casi al mismo ritmo se suceden declaraciones e informes del Ministro de Asuntos Exteriores, el Real Instituto Elcano, el Foro de Marcas Renombradas, etc. Sin ir más lejos, las encuestas elaboradas por el Barómetro del Real Instituto Elcano señalan que casi la mitad $(47,9 \%)$ de los menores de 45 años se han planteado emigrar, cifra que todavía es superior entre los menores de 30 años alcanzando el 67\%. La autoimagen de los españoles tampoco pasa por su mejor momento asociando y según el mismo estudio del Real Instituto Elcano más del $80 \%$ asocia España a la corrupción.

Estos son tan solo algunos aspectos en torno a los cuales se construye o "destruye" la imagen de marca España, aún siendo de manera involuntaria, pero que sí debe de ser tenido en cuenta a modo de análisis. No en vano, estamos hablando de una realidad líquida en la que una magnitud sólida como es la marca, sea de país o no (Sánchez Guitián, 2012: 17), se debe adaptar a las circunstancias. En este sentido y como veremos más adelante, intervienen muchos agentes, pero nos centraremos en lo que sigue en una de las actividades más visibles como es la campaña "I need Spain", encaminada a potenciar el país como destino turístico fidelizado desde una óptica alejada de la prototípica España de sol y playa. 


\section{Metodología}

Tal y como acabamos de mencionar, en el presente trabajo realizaremos un análisis descriptivo y crítico de la campaña "I need Spain" a través de sus distintas piezas y soportes, como spots audiovisuales, publicidad en medios impresos y las acciones digitales en términos de comunicación alternativa y redes sociales que básicamente han configurado la campaña turística de España por parte del gobierno en los últimos años (2010-13).

Para ello consideramos necesario definir y contextualizar el lugar que ocupa la Marca España en el contexto internacional, recurriendo a los principales estudios realizados a tales efectos. Consideramos los tres más relevantes, como son el Country Brand Index, el Anholt-Gfk Roper Nation Brands Index ${ }^{\mathrm{TM}}$ y el Bloom Consulting Country Brand Ranking (C)

Se trata, en esencia, de un trabajo que contextualiza el fenómeno de marca país, sus implicaciones y relevancia; todo ello aplicado al caso español a través de sus acciones comunicativas dirigidas frente a los mencionados aspectos coyunturales incontrolables por el emisor. Partimos de que si bien la campaña "I need Spain" está enfocada principalmente al turismo, también tiene efectos sobre otros elementos, eso sí, veremos como el turismo solo es una de las partes que evalúa la marca país en los estudios mencionados.

En lo que a estructura se refiere, hilaremos un discurso comenzando por consideraciones acerca de la gestión de la comunicación de marca, como ésta se aplica a la marca país, para pasar a continuación al caso español enunciado en el contexto de los estudios de marca país y finalizar con el análisis de la campaña y las conclusiones.

\section{La gestión de la marca}

Antes de profundizar en la gestión de la marca país y sus componentes, conviene establecer los paralelismos con la gestión de una marca comercial. Tal y como señala Sánchez Guitián (2012: 15) estamos inmersos más que nunca en una realidad líquida, en otras palabras, un entorno tecnológico y social cambiante al amparo de las nuevas tecnologías que marcan en gran parte los patrones del consumo, otrora opacos y azarosos para los grandes anunciantes. Tras esta idea obviamente subyace el principio "darwiniano" por excelencia, el de adaptarse o morir. Tanto es así, que la marca debe participar con el consumidor de sus conversaciones en un sentido amplio. Una marca no solo necesita poder evaluar o tan siquiera acceder a un feedback, sino que deberá generar los inputs necesarios de forma proactiva para poder superar los grandes retos del branding en la actualidad, como pueden ser la falta de diferenciación, la guerra de precios, la responsabilidad social corporativa o la decreciente fidelidad del cliente a una marca, entre otras. 
En este sentido, y tomando vocabulario de redes sociales como uno de los objetos de este estudio, el cliente potencial o real pasará a ser un fan o believer con una mayor comunicación e implicación con la marca que, a su vez, desembocará en la empatía que se trata de alcanzar. De ahí que marcas fuertes acaban por fortalecer la economía de un país que además cuenta con su propia imagen que se nutre de las anteriores (López Gil, 2012: 121), especialmente en lo referido a intangibles, que son aquellos aspectos que acaban por conferir el valor real de una marca - más allá de términos monetarios - para el consumidor y la sitúan en su mente, bien en la short list o bien como top-of-mind.

Así, en un contexto de cada vez mayor movilidad de personas e información, en definitiva, de globalización, también las marcas viajan alrededor del globo y no solo son consumidas por personas de distintas culturas, sino que un mismo país se compran productos y servicios mayoritariamente de origen extranjero, no siempre conocido o identificable. Es decir, las marcas también son en gran medida embajadoras como lo son todos aquellos valores y agentes que se asocian a un país y que veremos desglosados más adelante. En definitiva, no hay gran país ni gran imagen de marca país sin marcas significativas y relevantes en el contexto internacional (Cerviño y Rivera, 2007: 8).

Dicha tendencia ya se ha extendido a otras delimitaciones como puede ser la marca territorio o el propio city branding. Tomando esta evolución ya de por sí como justificación del presente trabajo por su relevancia y pertinencia, nos centraremos en lo que sigue en la imagen de marca país, desde su concepción teórico-práctica hasta los agentes que intervienen en su formación y difusión, así como en el caso español en particular a través de la marca España y una de sus últimas campañas.

\section{Las dimensiones de la imagen de marca país y su medición}

La imagen de marca país es un activo fundamental para defender los intereses de los Estados en las nuevas relaciones económicas y políticas internacionales (Sánchez Guitián; Zunzarren y Gorospe, 2013: 14). Es, o debería ser, percepción y proyección a partes iguales que implica a consumidores directos, indirectos, reales y potenciales (Valls, 1992: 7) con cuatro ejes principales como son el político, el comercial, el turístico y el cultural. El éxito de la imagen de país radica en gran medida en la fama y visibilidad con la que se exhibe frente a otros países. Como cualquier otra marca tiene un valor funcional, emocional e incluso económico. Partiendo, pues, de que la gestión de la marca país es un factor estratégico por todo lo señalado anteriormente, resulta clave poder medir su retorno. Para ello mencionaremos tres de los principales estudios de marca país, a saber, el Country Brand Index, el Anholt-Gfk Roper Nation Brands Index ${ }^{\mathrm{TM}}$ y el Bloom Consulting Country Brand Ranking (C).

Según el último Country Brand Index de Future Brand, España ocupa el $19^{\circ}$ lugar $(2012 / 2013)^{1}$, habiendo sufrido una pérdida de cinco puestos con respecto al registro 
anterior. Metodológicamente se trata de una investigación eminentemente cuantitativa a través de una encuesta a 3.500 hombres/mujeres de negocio y otro tipo de personas con viajes frecuentes de 14 países a la que se suman opiniones de expertos en turismo, exportaciones, inversiones y políticas públicas en 16 ciudades y la participación de una comunidad co-creativa en redes de crowdsourcing. Como base se utiliza el llamado sistema de decisión jerárquica (HDM) que se divide en siete apartados: el conocimiento acerca de la existencia del país (ese país se encontraría en la short list o incluso sería el top-of-mind), la familiaridad (como nivel superior al anterior en cuanto a conocimientos sobre los servicios que ofrece, por ejemplo), las asociaciones (qué cualidades se asocian al país, entre las que figuran calidad de vida, adecuación para negocios, cultura, turismo o sistema de valores), la preferencia o afinidad por el país, la consideración (como alternativa para viajar, invertir o consumir sus productos), la decisión (de visitar o invertir) y finalmente la recomendación familiares y amigos de visitar ese país o incluso considerarlo como posible país de residencia. Cabe reseñar aquí que se ha creado el acrónimo "PIIGS" en este estudio, grupo de países del que forma parte España junto a Portugal, Italia, Irlanda y Grecia; se trata de un grupo de naciones que comparten las consecuencias de la crisis económica, pero que gozan de grandes recursos naturales y culturales. De hecho, el propio informe recoge que no se ha producido una caída estrepitosa en términos de imagen, a pesar de lo que podría parecer a primera vista. Así, dos de ellos acompañan a España en el top 25, como son Italia e Irlanda. No obstante, la selección de siglas por sus similitud con un término inglés con asociaciones peyorativas parece cuanto menos llamativa.

El Anholt-Gfk Roper Nation Brands Index ${ }^{\mathrm{TM}}$, a su vez, es una evolución de Nation Brand Index elaborado por primera vez por Simon Anholt en 2005 para medir la imagen y reputación de las marcas país y su evolución en el tiempo. El Anholt-Gfk Roper Nation Brands Index ${ }^{\mathrm{TM} 2}$ se basa en 20.378 encuestas sobre 50 países que conforman el universo de dicho estudio. El hexágono de variables está compuesto por la exportación (determina la imagen de productos y servicios y hasta qué punto consumidores buscan o evitan de manera proactiva productos de cada país), la gobernanza (mide la opinión pública en cuanto a las competencias y justicia; describe creencias individuales acera del gobierno, así como su compromiso con temas globales como democracia, justicia, pobreza y el medio ambiente), la cultura y herencia (revela percepciones globales de la herencia cultural de cada país desde cine, música, arte, deportes y literatura), la población (mide la reputación de la población en cuanto a competencias, formación, apertura, amabilidad, etc. junto el nivel percibido de hostilidad y discriminación), el turismo (recoge el nivel de interés en visitar un país así como su atractivo natural y aquél creado por el hombre) y la inversión e inmigración (determina el poder de atraer a gente para vivir, trabajar o estudiar y revela como se percibe la situación económica y social). En este ranking España destaca por ser una de las naciones con mayores pérdidas de reputación, junto a Japón, pero sigue figurando en el top 20. 
Finalmente, el Bloom Consulting Country Brand Ranking (C) ${ }^{3}$ pretende responder a la pregunta de como un país se convierte en atractivo como fuente de bienestar y riqueza. El estudio se basa en tres grandes ejes, los negocios, el turismo y el talento. España destaca en el ranking de turismo ocupando el segundo lugar por detrás de Estados Unidos a nivel mundial y el primero en Europa. No obstante, este estudio ha recibido críticas por manejar ejes a priori antagónicos como son comunicar diversión y ocio al mismo tiempo que una mano de obra cualificada y trabajadora. Otro punto débil parece estribar en considerar por separado cada uno de los tres ejes. Sea como fuere, sirve para generar noticias positivas en torno a la imagen de España en medios nacionales e internacionales, lo cual, sin duda, es publicity bienvenida en términos de nation branding.

En resumen, observamos que existe mayoritariamente una metodología similar en todos estos estudios tendentes a utilizar encuestas y líderes de opinión para que se manifiesten sobre distintos aspectos en los que se basa el valor de la imagen de marca país. Bien es cierto que los resultados de España siguen siendo aceptables como uno de los 20 países con mejor reputación del mundo (la Organización de Naciones Unidas reconoce 192 países) y que incluso en algunos apartados como turismo o cultura la posición sería superior, pero hay que tener en cuenta que la tendencia es descendente por lo que se hace necesarios continuos esfuerzos de gestión y mejora de la imagen de marca país. A continuación analizaremos una de sus partes.

\section{La gestión de la marca país España}

El alto comisionado de la Marca España la define como una política de Estado, cuya eficacia reside en el largo plazo y que se basa en el consenso, por encima de cambios políticos ${ }^{4}$. Su objetivo consiste en mejorar la imagen de España, tanto en el interior como más allá de nuestras fronteras. A nadie se le escapa que España viene sufriendo con especial crudeza las consecuencias de la crisis económica desencadenada hace cinco años. Esto también se traduce en una pérdida de competitividad de sus marcas, tal y como se refleja en estudios como el Ranking de Fortune Global $500^{5}$, en el que se encuentran nueve empresas españolas, a saber, Banco Santander, Telefónica, Repsol YPF, BBVA, Iberdrola, Cepsa, Gas Natural Fenosa y Mapfre, mientras que la última edición del informe Best Global Brands $2012^{6}$ elaborado por Interbrand solo incluye a Zara y Banco Santander en los puestos 37 y 76 respectivamente.

Sería, no obstante, un error centrar únicamente nuestra atención en las marcas como caras visibles de un país. Así, se nombran los embajadores de la Marca España ${ }^{7}$ o Amigos de la Marca España ${ }^{8}$ con la concurrencia de Fernando Alonso (Deportes), Antonio Banderas (Cultura y Comunicación), Isak Andic (Gestión Empresarial), José Andrés (Turismo y Gastronomía), María Blasco (Ciencia e Innovación) la Organización Nacional de Trasplantes (Acción Social), Pedro Nueno (Relaciones Institucionales) y la Alta Velocidad Española (acreditación extraordinaria) en el primer grupo, 
mientras que en el segundo grupo figuran aquellas personalidades e instituciones de nacionalidad extranjera que han contribuido o apoyado significativamente en determinado país o territorio la imagen de España y/o de sus marcas renombradas.

$\mathrm{Y}$ es que no es simplemente una tarea del Gobierno gestionar la imagen de un país, sino que hay muchos más agentes implicados. Al margen de los mencionados, podemos ampliar la lista a Tourspain, Spaininfo, Instituto de Comercio Exterior (ICEX), el Instituto Cervantes, el Ministerio de Asuntos Exteriores, el Foro de Marcas Renombradas, la Asociación de Marcas Renombradas o la Oficina Española de Patentes y Marcas que a través de seminarios, talleres, presencia en ferias internacionales o campañas, como la que analizamos a continuación, trabajan en pos de la imagen de marca España.

\section{Análisis de la campaña "I need Spain"}

La campaña más reciente en los término señalados es "I need Spain" que Turespaña ha realizado tras el recorrido de "Spain is different" (de los años 60), la creación del logotipo de Miró (en los 80), "Spain marks" (2002) y "25 years going beyond the sun" (2009).

Con la pretensión de alcanzar un target más amplio y alejarse progresivamente la noción de sol y playa se desarrolló una estrategia especialmente centrada en medios digitales por parte de la agencia internacional McCann-Erickson. Dicha campaña fue fruto de un estudio que el Ministerio de Industria, Energía y Turismo realizó en el año 2009 y que evidenciaba la fuerte posición que España ocupaba en Europa como destino turístico, quedando otros aspectos en segundo plano. A ello se sumó la poca presencia del país en términos de notoriedad en el mercado asiático.

La campaña se ha lanzado en más de 40 países y se estima un alcance de más de 400 millones de ciudadanos, superando así las expectativas de una campaña lanzada anteriormente "Smile you are in Spain", cuyo target fue de 100 millones.

El objetivo de fondo era doble, por un lado, "reposicionar" a marca España en Europa y, por otro, posicionarla en mercados emergentes. Erik Alnas y Ale Burset, fotógrafos de prestigio internacional, y cineastas como Julio Medem contribuyeron a darle forma a la campaña en soportes gráficos, audiovisuales y online. Colaboraron entre muchos otros Ferrán Adriá, Gisela Pulido, el Real Madrid CF o los jugadores de las selecciones españolas de fútbol y baloncesto.

El objetivos de la campaña consistía en reflejar la diversidad de destinos y productos turísticos españoles a través de un amplio abanico de anuncios para medios impresos y audiovisuales. Por primera vez se incluyeron piezas exclusivamente pensadas y adaptadas al mercado asiático. Eso sí, todas las creatividades se basaron en el uso de elementos identificadores de España, como la gastronomía, el clima, las fiestas, la playa, el camino de Santiago, la cultura y un largo etcétera, sin olvidarnos de otras 
características inherentes al estilo de vida español como la siesta, el sol o la fiesta nocturna. A ello se sumó la moda o la innovación de diferentes ámbitos, aspectos en ocasiones menos visibles o asociados a España. Todo ello se presenta como experiencia y emoción, como valor intangible como sensación de España y de todo lo que significa o quiere representar en estos momentos y que la diferencia de otros países.

En junio de 2011 Turespaña lanza la nueva campaña de comunicación en medios digitales como una apuesta innovadora por el turismo con el fin de reforzar la marca turística España en los mercados internacionales con el fin de posicionarla como destino líder.

El eje de esta estrategia se basa en que España es el primer destino del mundo con mayor fidelización por parte de los turistas; de los 53 millones de turistas extranjeros que visitan España, un $85 \%$ vuelve. Dicha idea es recogida en las redes sociales donde se buscan a los auténticos Spain addicts, para que se conviertan en embajadores de la marca España y la propaguen a través de sus cuentas y experiencias. Para ello se crea la plataforma www.ineedspain.com donde se pueden compartir las vivencias en fotos, vídeos y mensajes. De esta manera, los testimonios de personajes de renombre se suman a las de personas anónimas que han visitado el país y han querido dejar constancia de su experiencia. La iniciativa pretende involucrar al turista fiel y generar más empatía, en definitiva, conversación con la marca.

La página arranca con la invitación al registro y presenta una apariencia muy visual y dinámica casi a modo de blog. Contiene vídeos y declaraciones y juega con los colores. Tiene una configuración en forma de mosaico, uniendo fotografías, vídeos y noticias, como un Tumblr, con muchas ciudades y pueblos de España. Se incluyen declaraciones de figuras relevantes de cada cuidad sobre los sitios que más les gustan de cada lugar.

Tal y como ya mencionamos más arriba, la lista de famosos participantes es larga. Destacan los jugadores del Real Madrid CF, como por ejemplo Cristiano Ronaldo, Xabi Alonso, Karim Benzema o Sergio Ramos, que protagonizan un vídeo de promoción turística de la capital y de España que se emitió en las televisiones nacionales. También son embajadores de la marca España, los motociclistas Jorge Lorenzo, Marc Márquez y Toni Elías, además del chef Ferrán Adriá, quien ya colaboró de forma desinteresada en la campaña para medios convencionales.

Los vídeos son temáticos de modo que los jugadores del Real Madrid CF van explicando qué les gusta de la ciudad donde juegan casi a modo de guía turístico, pero con su toque personal. No en vano, son futbolistas de distinta procedencia, tanto nacional como internacional. Esta campaña se ha lanzado en países como Alemania, Francia, Italia y Reino Unido. En una primera fase se podía ver en Yahoo!, Google, Youtube y en webs de viajes, y posteriormente estuvo presente en los medios digitales de estos países.

Los resultados ${ }^{10}$ son bastante ambiguos ya que prevalece el sentimiento de "neutralidad" frente al de positivismo, aun así no se registra ninguna percepción negativa por 
lo que los resultados son óptimos. En cuanto a palabras más utilizadas se registra en el primer puesto Spain; a continuación campaña seguida de Spanish entre otras.

La presencia en redes sociales se intentó reforzar con la creación de un perfil en Facebook. La página fue puesta en marcha el 5 de marzo de 2010 y en 2013 apenas supera los 8.000 usuarios, datos que no invitan al optimismo, al igual que un elevado número de comentarios negativos (más que positivos en todo caso). La cuenta de Twitter cuenta con 26.241 seguidores y su canal de Youtube 5.105 suscripciones. En todo caso, son datos bastante humildes con respecto a otras iniciativas. Como acción complementaria en el 2013 Turespaña echa mano de formatos publicitarios alternativos con una acción de marketing de guerrilla en un aeropuerto donde se lleva a un grupo de músicos a interpretar "Adiós con el corazón" para los viajeros a los que se les va entrevistando y recogiendo sus testimonios. La idea consistía en realizar una promoción viral, apelando a la sencillez, la austeridad y el recorrido natural de "compartir" que tienen las estrategias de comunicación alternativa.

\section{Conclusiones}

No cabe la menor duda de que la campaña analizada en este trabajo tiende a afianzar la marca país y es una de las bases sobre las que trabajar para seguir gestionando ese intangible nacional. Sirven de punto de apoyo para configurar una imagen a nivel internacional. Sin embargo, queda patente el elevado grado de complejidad de la gestión de la misma por la concurrencia de factores incontrolables como todos aquellos derivados de la coyuntura económica, la política o la imagen que se proyecta a nivel internacional del país. Son demasiados los agentes que de forma voluntaria e involuntaria intervienen en la creación y mantenimiento o empeoramiento de la imagen de marca país. Mientras Morgan Stanley recientemente alentaba a modo de grito con un "viva España", otros indicadores no invitan a tanto optimismo, mientras que el país se debate a nivel interno en varios frentes políticos: la grave crisis económica, la elevada tasa de paro, cuestiones de soberanías e independencias y los múltiples casos de corrupción que salpican a políticos y a aquellos que no lo son, por no hablar de la baja popularidad de los miembros de la Casa Real, otrora considerada la mejor embajadora de España. He aquí uno de los principales problemas: la imagen que tienen los españoles de su propio país que dista mucho de la impresión que se genera en el extranjero. Es decir, el esfuerzo comunicativo también tiene que ser interno, tiene que ser de convicción o, mejor dicho, de autoconvicción.

Como estrategia publicitaria y posterior elaboración y diseminación de piezas, la idea de buscar la conversación y empatía nos parece acertada, si bien consideramos que necesita refuerzo para no diluirse o quedar obsoleta. Nuestras caras más visibles en todos los niveles deben seguir la senda emprendida, sean embajadores o amigos de la marca España, porque al final la pretensión última es que el ciudadano español también lo sea y predique con el ejemplo. 
Casi a modo de manual cabría pedir la implicación de las administraciones públicas, las empresas, los medios de comunicación y los propios ciudadanos, pero resulta casi utópico. Eso sí, consideramos que con la creación del Alto Comisionado para la Marca España se ha dado en su momento un paso en la dirección correcta y somos conscientes de la dificultad de armonizar actividades conjuntas, ya no solo desde el ámbito de la comunicación.

Finalmente y sin pretender criticar la labor desempeñada por los responsables directos de la gestión de la marca España, cabría preguntarse por qué no orientarse en aquellos países que según los índices mencionados parecen haber hecho bien las cosas y entre los que figuran vecinos como Suiza o los países escandinavos, junto con Nueva Zelanda y Canadá. Somos conscientes de que son realidades e idiosincrasias bien distintas, pero al menos habría que plantearse las ideas de fondo siempre y cuando haya disposición a invertir en la marca España como un activo que beneficia a un país y a todos los que formamos parte de él en términos de prestigio y reconocimiento.

\section{Bibliografía}

Annual ranking of the world's largest corporations 2011. Fortune. Disponible en : http://money.cnn.com/magazines/fortune/global500/2011/countries/Spain.html. [13/01/2013]

Best Global Brands 2012. Interbrand. Disponible en: http://www.interbrand.com/es/ best-global-brands/2012/downloads.aspx. [25/07/2013]

BIGNÉ, E. (2000). "Image and Spanish Country of Origin Effect". En: CANNON, J., WARNER, R. \& ODBER DE BAUBETA, P.A. (eds.). Advertising and Identity in Europe. The I of the Beholder. Bristol: Editorial Intellect. P. 5-17.

BONET FERRER, J.L. (2011). "El foro de marcas renombradas españolas, una experiencia para la competitividad internacional". Disponible en: http://www.marcasrenombradas.com/wp-content/uploads/2011/12/JL-BONET-Econom\%C3\%ADa-Industrial.pdf. [13/08/2013]

Brand Finance Nation Brands 100. Disponible en: http://brandfinance.com/images/ upload/bfnb_100_2011_web_sp.pdf. [20/09/2013]

CAPRIOTTI, P. (2009). Branding corporartivo. Fundamentos para la gestión estratégica de la identidad corporativa. Santiago de Chile: Colección de libros de la empresa.

CERVIÑO, J. \& BAENA, V. (2011). "La globalización de las marcas la competitividad. Tendencias y retos para las empresas españolas." Disponible en: http:// www.minetur.gob.es/Publicaciones/Publicacionesperiodicas/EconomiaIndustrial/RevistaEconomiaIndustrial/379/Julio\%20Cervi\%C3\%B10\%20Web\%20 EI\%20379.pdf. [9/11/ 2012]

Country Brand Index 2012/2013. Future Brand. Disponible en: http://www.futurebrand.com/images/uploads/studies/cbi/CBI_2012-Final.pdf. [20/ 09/2013] 
ECHEVERRI, M. (2009). (Resumen de ponencia publicada en ASCOLFA 2009) y recogida en el blog "Red de Marketing". Disponible en: http://redmkt.wordpress. com/2009/05/08/la-marca-pais-el-reflejo-de-la-identidad-cultural-y-empresarialde-una-nacion/. [21/07/2013]

Foro de Marcas Españolas Renombradas (2011). "Memoria X Aniversario 19992009". Disponible en: http://www.marcasrenombradas.com/wp-content/ uploads/2011/08/593-ANEXO-11.pdf. [24/04/2013]

LÓPEZ GIL, P. (2012). "Las marcas, un pilar fundamental de la imagen país." En: Retos de nuestra acción exterior: Diplomacia pública y Marca España. P. 119-139.

Made in Spain = Hecho en España: la imagen de España y sus marcas en el mundo (2003). Foro de Marcas Renombradas Españolas.

“Marcas. Branding que es gerundio”. En: Mejores Marcas Españolas 2011. Interbrand. 2011. Disponible en: http://www.interbrand.com/Libraries/Branding_Studies_ES/ MEJORES_MARCAS_ESPA\%C3\%91OLAS_2011.sflb.ashx. [03/09/2013]

PERALBA, R. \& CERVIÑNO, J. (2007). “Marca ‘España’, ¿para qué?”. En: Cinco Días, 28/08/2007. P. 11.

PERALBA, R. (2009). El posicionamiento estratégico de la marca España. Madrid: Círculo de Empresarios.

SÁNCHEZ GUITIÁN, J.M. (2012). Marca país. España, una marca líquida. Madrid: ESIC.

SÁNCHEZ GUITIÁN, J.M.; ZUNZARREN, H. \& GOROSPE, B. (2013). ¿Cómo se gestiona una marca país? Madrid: ESIC.

VALLS, J.F. (1992). La imagen de marca de los países. Madrid: McGraw-Hill.

\section{Notas}

1 Country Brand Index 2012/2013. Future Brand. Disponible en: http://www.futurebrand.com/images/ uploads/studies/cbi/CBI_2012-Final.pdf . [20/09/2013]

2 Anholt-Gfk Roper Nation Brands Index ${ }^{\mathrm{TM}}$. Disponible en: http://www.gfk.com/news-and-events/ press-room/press-releases/pages/two-thirds-of-nations-experience-reputation-decline-in-2012-nation-brands-index.aspx [22/09/2013]

3 Bloom Consulting Country Brand Ranking (C. Disponible en: http://bloom-consulting.com/en/country-brands-ranking [15/10/2013]

4 Qué es Marca España. Disponible en: http://marcaespana.es/es/quienes-somos/que-es-marca-espana. php [24/019/2013]

5 Annual ranking of the world's largest corporations 2011. Fortune. Disponible en : http://money.cnn. com/magazines/fortune/global500/2011/countries/Spain.html. [13/01/2013]

6 Best Global Brands 2012. Interbrand. Disponible en: http://www.interbrand.com/es/best-globalbrands/2012/downloads.aspx. [25/07/2013]

7 Embajadores de la Marca España 2013. Disponible en: http://www.marcasrenombradas.com/ehme/. $[10 / 09 / 2013]$ 
8 Amigos de la Marca España. Disponible en: http://www.marcasrenombradas.com/seccion/ amigos-marca-espana/. [10/09/2013]

9 Disponible en: http://www.ineedspain.com/?lang=es. [3/09/2013]

10 Según indicadores obtenidos en http://www.socialmention.com [15/07/2013]

\section{Los autores}

Juan Manuel Corbacho Valencia es Licenciado en Publicidad y Relaciones Públicas y Traducción e Interpretación por la Universidad de Vigo. Realizó su tesis sobre publicidad internacional, materia que imparte en la Facultad de Ciencias Sociales y de la Comunicación (Universidad de Vigo - Campus de Pontevedra) de la que en el momento de la publicación es decano tras pasar por otros cargos académicos como el de vicedecano de relaciones internacionales. Junto con su línea de investigación de comunicación publicitaria global, también ha publicado trabajos en el ámbito de las relaciones públicas y protocolo. Ha realizado estancias de investigación en Brasil, Chile, México, Portugal y Alemania.

Mónica Valderrama es profesora Contratada Doctora de la Universidad de Vigo del Área de Comunicación Audiovisual y Publicidad. Licenciada en Periodismo por la Universidad de Santiago de Compostela (1996), Licenciada en Publicidad y RR.PP. por la Universidad de Vigo (1998), Doctora en Publicidad y RR.PP. por la Universidad de Vigo (2003) y Premio Extraordinario Doctorado. Experiencia profesional en diferentes medios de comunicación. Autora de A publicidade televisiva en Galicia. Análise dos emisores, soportes e tendencias creativas (1960-2000), CGAI, 2008. Ha realizado estancias en centros extranjeros en México, Brasil e Italia en las Universidades Anahuac (campus Xalapa y Cancún, 2007 y 2009), PUC-Minas Gerais (2000), Colima (2001 y 2002) y Lumsa (Roma, 2010, 11 y 12). Líneas de investigación: tendencias creativas en medios audiovisuales emergentes, evolución de la producción publicitaria televisiva, nuevos formatos publicitarios audiovisuales, identidades televisivas, y nuevas tecnologías aplicadas a la comunicación organizacional.

Silvia García Mirón es profesora ayudante del Departamento de Comunicación Audiovisual y Publicidad de la Universidade de Vigo. Licenciada en Publicidad y Relaciones Públicas (2006) y Comunicación Audiovisual (2009) por la Universidade de Vigo. Entre sus principales líneas de investigación destacan el análisis de estrategias de programación de las emisoras televisas; el estudio de nuevas técnicas de fidelización del telespectador; la planificación y planteamiento de estrategias publicitarias en nuevos medios; y el uso y aplicación de las NTICs en el ámbito docente. Desarrollando, actualmente, su tesis doctoral centrada en un análisis sobre los contenidos y las estrategias de programación en prime time de Antena 3 Televisión, la primera emisora televisiva española. 